Kragujevac Journal of Mathematics

Volume 38(1) (2014), Pages 51-71.

\title{
ABSTRACT MULTI-TERM FRACTIONAL DIFFERENTIAL EQUATIONS
}

\author{
C.-G. $\mathrm{LI}^{1}$, M. KOSTIĆ${ }^{2}$, AND M. $\mathrm{LI}^{3}$
}

\begin{abstract}
The present paper is an addendum to our recent work on abstract time-fractional equations of the following form:

$$
\begin{aligned}
& \mathbf{D}_{t}^{\alpha_{n}} u(t)+\sum_{i=1}^{n-1} A_{i} \mathbf{D}_{t}^{\alpha_{i}} u(t)=A \mathbf{D}_{t}^{\alpha} u(t)+f(t), \quad t>0, \\
& u^{(k)}(0)=u_{k}, \quad k=0, \cdots,\left\lceil\alpha_{n}\right\rceil-1,
\end{aligned}
$$

where $n \in \mathbb{N} \backslash\{1\}, A$ and $A_{1}, \cdots, A_{n-1}$ are closed linear operators on a sequentially complete locally convex space $X, 0 \leq \alpha_{1}<\cdots<\alpha_{n}, 0 \leq \alpha<\alpha_{n}, f(t)$ is an $X$-valued function, and $\mathbf{D}_{t}^{\alpha}$ denotes the Caputo fractional derivative of order $\alpha$ [2]. We analyze the existence and uniqueness of solutions of the equation (0.1), and prove a Ljubich type uniqueness theorem in this context.
\end{abstract}

\section{IntRoduction AND PRELIMINARIES}

During the past three decades or so, there has been an explosion of interest in fractional differential equations and fractional dynamics, primarily from their invaluable importance in modeling of various phenomena appearing in physics, chemistry, mathematical biology and engineering. After significant research of E. Bazhlekova [2], it became clear that the abstract Volterra integro-differential equations provide a general framework for the analysis of a large class of abstract time-fractional equations with Caputo derivatives. For more details about fractional differential equations and Volterra integro-differential equations, the reader may consult the monographs by K. Diethelm [8], V. Kiryakova [13] and K. S. Miller-B. Ross, I. Podlubny, J. Prüss [28]-[30].

Key words and phrases. Abstract time-fractional equations, $k$-regularized $\left(C_{1}, C_{2}\right)$-existence and uniqueness families, Abstract Volterra equations.

2010 Mathematics Subject Classification. Primary: 47D06, 47D62. Secondary: 47D60, 47D99.

Received: March 15, 2013. 
In this paper, we continue the analysis of the abstract multi-term fractional differential equation (0.1); cf. also [2]-[4] and [14]-[27]. The most important subcase of $(0.1)$ is, without any doubt, the abstract Cauchy problem $\left(A C P_{n}\right)$ :

$$
\left(A C P_{n}\right):\left\{\begin{array}{l}
u^{(n)}(t)+A_{n-1} u^{(n-1)}(t)+\cdots+A_{1} u^{\prime}(t)+A_{0} u(t)=0, t \geq 0, \\
u^{(k)}(0)=u_{k}, k=0, \cdots, n-1
\end{array}\right.
$$

whose qualitative properties have been studied in a series of papers by T.-J. Xiao and J. Liang (cf. the monograph [31] for a comprehensive exposition of results). As some other very important subcases of (0.1), we quote the abstract Basset-BoussinesqOseen equation

$$
u^{\prime}(t)-A \mathbf{D}_{t}^{\alpha} u(t)+u(t)=f(t), \quad t \geq 0 \quad(\alpha \in(0,1)),
$$

which describes the unsteady motion of a particle accelerating in a viscous fluid under the action of the gravity, and the equation

$$
u^{\prime \prime}(t)+A \mathbf{D}_{t}^{\alpha} u(t)+u(t)=f(t), t \geq 0 \quad(\alpha \in(1,2)),
$$

which models an oscillation process with fractional damping term. The study of equations (1.1)-(1.2) has been initiated by C. Lizama in his joint papers with H. Prado [26] and A. Karczewska [27] (cf. [19] and [22] for more details on the $C$-wellposedness of equations (1.1)-(1.2) in some classes of Banach or Fréchet spaces). Of importance is also to stress that H. Jiang, F. Liu, I. Turner and K. Burrage have recently analyzed in [11] the following scalar multi-term time-space Caputo-Riesz fractional advection diffusion equation

$$
\mathbf{D}_{t}^{\alpha_{n}} u(t, x)+\sum_{j=1}^{n-1} c_{j} \mathbf{D}_{t}^{\alpha_{j}} u(t, x)=k_{\beta} \frac{\partial^{\beta} u(t, x)}{\partial|x|^{\beta}}+k_{\gamma} \frac{\partial^{\gamma} u(t, x)}{\partial|x|^{\gamma}},
$$

for $t \geq 0,0 \leq x \leq L$, where $n \in \mathbb{N} \backslash\{1\}, c_{1}, \cdots, c_{n-1} \in \mathbb{C}, 0 \leq \alpha_{1}<\cdots<$ $\alpha_{n} \leq 2,0<\beta<1,1<\gamma \leq 2, k_{\beta}, k_{\gamma}>0, L>0$ and $\frac{\partial^{\beta} u(t, x)}{\partial|x|^{\beta}}$ denotes the Riesz fractional operator of order $\beta$ (cf. [5] for various evolution models of equation (1.3) and its backwards analogue); concerning abstract (multi-term) fractional differential equations involving Riemann-Liouville or Liouville-Grünwald fractional derivatives, the reader may consult [3], [9]-[10], [12] and [25]. Finally, there is no need to say that it would be really difficult to include all the relevant subcases of $(0.1)$ not mentioned so far; thus we will refer the reader to [15] and the references cited there for further information on the subject.

The organization of paper is as follows. In the first part of Section 2, we shall recall some known definitions and assertions necessary for our further work [20]. In Theorem 2.1-Theorem 2.2 and Remark 2.1, we investigate mutual relations between $k$-regularized $\left(C_{1}, C_{2}\right)$-existence and uniqueness propagation families ( $k$-regularized $C$-resolvent propagation families) and $k$-regularized $\left(C_{1}, C_{2}\right)$-existence and uniqueness families ( $k$-regularized $C$-resolvent families). The analysis of these relations is very complicated in the general case, so that we must focus our attention on the 
case in which $A_{j}=c_{j} I$ for all $i \in \mathbb{N}_{n-1}$. If so, then we will be able to express any single operator family $\left(R_{i}(t)\right)_{t \in[0, \tau)}$ of a locally equicontinuous $k$-regularized $C$ resolvent propagation family $\left(\left(R_{0}(t)\right)_{t \in[0, \tau)}, \cdots,\left(R_{m_{n}-1}(t)\right)_{t \in[0, \tau)}\right)$ for $(0.1)$ in terms of $\left(R_{0}(t)\right)_{t \in[0, \tau)}$ (cf. Remark 2.1(ii), and [31, pp. 116-119] for a slightly different approach in the case of the abstract Cauchy problem $\left.\left(A C P_{n}\right)\right)$. Further on, we would like to note that perturbations of existence and uniqueness families for the abstract Cauchy problem (0.1) have not been well-studied in the corresponding literature [6-7, 20, 23-24, 33-34], even in the case of equations of integer order. In Theorem 2.3, we transfer results of R. deLaubenfels [7, Section VI] to existence and uniqueness families for the equation (0.1); as an application, we consider in Example 2.1 bounded perturbations of a damped Klein-Gordon equation in $L^{p}$-spaces [33, 20]. Notice also that T.-J. Xiao and J. Liang [32] have considered Desch-Schappacher multiplicative and additive perturbation type theorems for $C_{1}$-existence families for the abstract Cauchy problem $u^{(n)}(t)=A u(t)(t \geq 0) ; u^{(k)}(0)=u_{k}(0 \leq k \leq n)$, in the setting of Banach spaces. We shall examine the possibility of extension of their results to the abstract multi-term fractional differential equations in a separate paper. The existence and uniqueness of solutions of the equation (0.1) are analyzed in the third section of paper.

Unless stated otherwise, $X$ denotes a Hausdorff sequentially complete locally convex space over the field of complex numbers, SCLCS for short, and the abbreviation $\circledast_{X}$, or simply $\circledast$, stands for the fundamental system of seminorms which defines the topology of $X$. By $L(X)$ we denote the space of all continuous linear mappings from $X$ into $X$. Let $\mathcal{B}$ be the family of bounded subsets of $E$ and let $p_{B}(T):=\sup _{x \in B} p(T x), p \in \circledast$, $B \in \mathcal{B}, T \in L(X)$. Then $p_{B}(\cdot)$ is a seminorm on $L(X)$ and the system $\left(p_{B}\right)_{(p, B) \in \circledast \times \mathcal{B}}$ induces the Hausdorff locally convex topology on $L(X)$. Henceforth $A$ denotes a closed linear operator acting on $X, C \in L(X)$ is an injective operator, and the convolution like mapping $*$ is given by $f * g(t):=\int_{0}^{t} f(t-s) g(s) d s$. The domain, resolvent set, range, point spectrum and adjoint operator of $A$ are denoted by $D(A), \rho(A), R(A)$, $\sigma_{p}(A)$ and $A^{*}$, respectively. Since no confusion seems likely, we will identify $A$ with its graph. Recall that the $C$-resolvent set of $A$, denoted by $\rho_{C}(A)$, is defined by

$$
\rho_{C}(A):=\left\{\lambda \in \mathbb{C} ; \lambda-A \text { is injective and }(\lambda-A)^{-1} C \in L(X)\right\} .
$$

Suppose now that $Y$ is also a sequentially complete locally convex space over the field of complex numbers. Then we denote by $L(Y, X)$ the space which consists of all bounded linear operators from $Y$ into $X$. By $\circledast_{Y}$ and $I$ we denote the fundamental system of seminorms which defines the topology on $Y$, and the identity operator on $X$, respectively. If $0<\tau \leq \infty$, then a strongly continuous operator family $(W(t))_{t \in[0, \tau)} \subseteq$ $L(Y, X)$ is said to be locally equicontinuous iff, for every $T \in(0, \tau)$ and for every $p \in \circledast_{X}$, there exist $q_{p} \in \circledast_{Y}$ and $c_{p}>0$ such that $p(W(t) y) \leq c_{p} q_{p}(y), y \in Y$, $t \in[0, T]$.

Given $s \in \mathbb{R}$ in advance, set $\lfloor s\rfloor:=\sup \{l \in \mathbb{Z}: s \geq l\}$ and $\lceil s\rceil:=\inf \{l \in \mathbb{Z}: s \leq l\}$. The Gamma function is denoted by $\Gamma(\cdot)$ and the principal branch is always used to 
take the powers. Set $\mathbb{N}_{l}:=\{1, \cdots, l\}, \mathbb{N}_{l}^{0}:=\{0,1, \cdots, l\}, 0^{\zeta}:=0, g_{\zeta}(t):=t^{\zeta-1} / \Gamma(\zeta)$ $(\zeta>0, t>0)$ and $g_{0}:=$ the Dirac $\delta$-distribution; the symbol $\delta_{k l}$ denotes the Kronecker delta. If $\omega>0$, then we say that a function $f:(\omega, \infty) \rightarrow X$ belongs to the class $L T-X$, if there exists a function $h(\cdot) \in C([0, \infty): X)$ such that, for every $p \in \circledast$, there exists $M_{p}>0$ satisfying $p(h(t)) \leq M_{p} e^{\omega t}, t \geq 0$, and $f(t)=\int_{0}^{\infty} e^{-\lambda t} h(t) d t, \quad \lambda>\omega$. We assume henceforth that $A$ and $A_{1}, \cdots, A_{n-1}$ are closed linear operators on $X$ as well as that $0 \leq \alpha_{1}<\cdots<\alpha_{n}$ and $0 \leq \alpha<\alpha_{n}$. Set $m_{j}:=\left\lceil\alpha_{j}\right\rceil, 1 \leq j \leq n$, $m:=m_{0}:=\lceil\alpha\rceil, A_{0}:=A$ and $\alpha_{0}:=\alpha$.

Let $\alpha>0$, let $\beta \in \mathbb{R}$, and let the Mittag-Leffler function $E_{\alpha, \beta}(z)$ be defined by $E_{\alpha, \beta}(z):=\sum_{n=0}^{\infty} z^{n} / \Gamma(\alpha n+\beta), z \in \mathbb{C}$; here we assume that $1 / \Gamma(\alpha n+\beta)=0$ if $\alpha n+\beta \in-\mathbb{N}_{0}$. Set, for short, $E_{\alpha}(z):=E_{\alpha, 1}(z), z \in \mathbb{C}$. Then we define the Wright function $\Phi_{\gamma}(t)$ by $\Phi_{\gamma}(t):=\mathcal{L}^{-1}\left(E_{\gamma}(-\lambda)\right)(t), t \geq 0$, where $\mathcal{L}^{-1}$ denotes the inverse Laplace transform (cf. [2, Section 1.3] for more details about the Mittag-Leffler and Wright functions).

Suppose $0<\tau \leq \infty, k \in C([0, \tau)), k \neq 0, a \in L_{l o c}^{1}([0, \tau))$ and $a \neq 0$. We refer the reader to [17]-[20] and [23] for the notions of various types of (local) $(a, k)$-regularized $\left(C_{1}, C_{2}\right)$-existence and uniqueness families, $(a, k)$-regularized $C$-resolvent families and their subgenerators.

\section{Further Results on the $C$-Wellposedness of $(0.1)$}

The notions of strong solutions of the abstract Cauchy problem (0.1), and its $C$ wellposedness, are understood in the sense of [20, Definition 2.1]. If $u(t) \equiv u\left(t ; u_{0}, \cdots\right.$ $\left.\cdot, u_{m_{n}-1}\right), t \geq 0$ is a strong solution of $(0.1)$, with $f(t) \equiv 0$ and initial values $u_{0}, \cdot$ $\cdot, u_{m_{n}-1} \in R(C)$, then we can integrate both sides of (0.1) $\alpha_{n}$-times, and make use of the equality $[2,(1.21)]$. In such a way, we get that the function $u(t), t \geq 0$ satisfies the following integral equation:

$$
\begin{aligned}
u(\cdot) & -\sum_{k=0}^{m_{n}-1} u_{k} g_{k+1}(\cdot)+\sum_{j=1}^{n-1} g_{\alpha_{n}-\alpha_{j}} * A_{j}\left[u(\cdot)-\sum_{k=0}^{m_{j}-1} u_{k} g_{k+1}(\cdot)\right] \\
& =g_{\alpha_{n}-\alpha} * A\left[u(\cdot)-\sum_{k=0}^{m-1} u_{k} g_{k+1}(\cdot)\right] .
\end{aligned}
$$

The following definition can be compared with Definition 2.2 of [21].

Definition 2.1. Suppose $0<\tau \leq \infty, k \in C([0, \tau)), C_{1} \in L(Y, X)$, and $C_{2} \in L(X)$ is injective.

(i) A strongly continuous operator family $(E(t))_{t \in[0, \tau)} \subseteq L(Y, X)$ is said to be a (local, if $\tau<\infty)\left(k, C_{1}\right)$-existence family for $(0.1)$ iff, for every $y \in Y$ and $t \in[0, \tau)$, the following holds: $A_{j}\left(g_{\alpha_{n}-\alpha_{j}} * E\right)(\cdot) y \in C([0, \tau): X)$ for $0 \leq j \leq n-1$, and 


$$
E(t) y+\sum_{j=1}^{n-1} A_{j}\left(g_{\alpha_{n}-\alpha_{j}} * E\right)(t) y-A\left(g_{\alpha_{n}-\alpha} * E\right)(t) y=k(t) C_{1} y .
$$

(ii) A strongly continuous operator family $(U(t))_{t \in[0, \tau)} \subseteq L(X)$ is said to be a (local, if $\tau<\infty)\left(k, C_{2}\right)$-uniqueness family for (0.1) iff, for every $t \in[0, \tau)$ and $x \in \bigcap_{0 \leq j \leq n-1} D\left(A_{j}\right)$, the following holds:

$$
U(t) x+\sum_{j=1}^{n-1}\left(g_{\alpha_{n}-\alpha_{j}} * U(\cdot) A_{j} x\right)(t)-\left(g_{\alpha_{n}-\alpha} * U(\cdot) A x\right)(t) x=k(t) C_{2} x .
$$

(iii) Suppose $X=Y$. Then a strongly continuous family $\left((E(t))_{t \in[0, \tau)},(U(t))_{t \in[0, \tau)}\right)$ $\subseteq L(X) \times L(X)$ is said to be a (local, if $\tau<\infty)\left(k, C_{1}, C_{2}\right)$-existence and uniqueness family for $(0.1)$ iff $(E(t))_{t \in[0, \tau)}$ is a $\left(k, C_{1}\right)$-existence family for $(0.1)$, and $(U(t))_{t \in[0, \tau)}$ is a $\left(k, C_{2}\right)$-uniqueness family for $(0.1)$.

(iv) Suppose $C=C_{1}=C_{2}$. Then a strongly continuous operator family $(R(t))_{t \in[0, \tau)}$ $\subseteq L(X)$ is said to be a (local, if $\tau<\infty)(k, C)$-resolvent family for $(0.1)$ iff $(R(t))_{t \in[0, \tau)}$ is a $(k, C)$-uniqueness family for $(0.1), R(t) A_{j} \subseteq A_{j} R(t)$, for $0 \leq j \leq n-1$ and $t \in[0, \tau)$, as well as $R(t) C=C R(t), t \in[0, \tau)$, and $C A_{j} \subseteq A_{j} C$, for $0 \leq j \leq n-1$.

The notion of (exponential) analyticity of various types of $\left(C_{1}, C_{2}\right)$-existence and uniqueness families for (0.1) is taken in the sense of [20, Definition 1.2(ii)]. We refer the reader to [20, Definition 3.1] for the notions of $k$-regularized $\left(C_{1}, C_{2}\right)$-existence and uniqueness families and $k$-regularized $C$-resolvent families for $(0.1)$; if $k(t)=1$, $t \in[0, \tau)$, then any such operator family is simply called a $\left(C_{1}, C_{2}\right)$-existence and uniqueness family for (0.1) ( $C$-resolvent family for $(0.1))$. Notice that the following assertions hold:

(i) Suppose $(E(t))_{t \in[0, \tau)} \subseteq L(Y, X)$ is a strongly continuous operator family. Then $(E(t))_{t \in[0, \tau)}$ is a (local) $\left(k, C_{1}\right)$-existence family for $(0.1)$ iff $\left(\left(g_{m_{n}-1} * E\right)(t)\right)_{t \in[0, \tau)}$ is a $k$-regularized $C_{1}$-existence family for $(0.1)$.

(ii) Suppose $(U(t))_{t \in[0, \tau)} \subseteq L(X)$ is a strongly continuous operator family. Then $(U(t))_{t \in[0, \tau)}$ is a (local) $\left(k * g_{m_{n}-1}, C_{2}\right)$-uniqueness family for $(0.1)$ iff $(U(t))_{t \in[0, \tau)}$ is a (local) $k$-regularized $C_{2}$-uniqueness family for $(0.1)$.

The reader may consult [20, Definition 2.7] for the notions of strong (mild) solutions of the following inhomogeneous Volterra equation:

$$
u(t)+\sum_{j=1}^{n-1}\left(g_{\alpha_{n}-\alpha_{j}} * A_{j} u\right)(t)=f(t)+\left(g_{\alpha_{n}-\alpha} * A u\right)(t), \quad t \in[0, T] .
$$

One can similarly define the notion of a strong (mild) solution of the problem (2.1).

Given $i \in \mathbb{N}_{m_{n}-1}^{0}$ in advance, set $D_{i}:=\left\{j \in \mathbb{N}_{n-1}: m_{j}-1 \geq i\right\}$. 
Definition 2.2. Suppose $0<\tau \leq \infty, k \in C([0, \tau)), C, C_{1}, C_{2} \in L(X), C$ and $C_{2}$ are injective. A sequence $\left(\left(R_{0}(t)\right)_{t \in[0, \tau)}, \cdots,\left(R_{m_{n}-1}(t)\right)_{t \in[0, \tau)}\right)$ of strongly continuous operator families in $L(X)$ is called a (local, if $\tau<\infty)$ :

(i) $k$-regularized $C_{1}$-existence propagation family for $(0.1)$ iff $R_{i}(0)=\left(k * g_{i}\right)(0) C_{1}$ and the following holds:

$$
\begin{aligned}
& {\left[R_{i}(\cdot) x-\left(k * g_{i}\right)(\cdot) C_{1} x\right]+\sum_{j \in D_{i}} A_{j}\left[g_{\alpha_{n}-\alpha_{j}} *\left(R_{i}(\cdot) x-\left(k * g_{i}\right)(\cdot) C_{1} x\right)\right]} \\
& +\sum_{j \in \mathbb{N}_{n-1} \backslash D_{i}} A_{j}\left(g_{\alpha_{n}-\alpha_{j}} * R_{i}\right)(\cdot) x \\
& = \begin{cases}A\left(g_{\alpha_{n}-\alpha} * R_{i}\right)(\cdot) x, & m-1<i, x \in X, \\
A\left[g_{\alpha_{n}-\alpha} *\left(R_{i}(\cdot) x-\left(k * g_{i}\right)(\cdot) C_{1} x\right)\right](\cdot), & m-1 \geq i, x \in X,\end{cases}
\end{aligned}
$$

for any $i=0, \cdots, m_{n}-1$.

(ii) $k$-regularized $C_{2}$-uniqueness propagation family for $(0.1)$ iff $R_{i}(0)=(k *$ $\left.g_{i}\right)(0) C_{2}$ and

$$
\begin{aligned}
& {\left[R_{i}(\cdot) x-\left(k * g_{i}\right)(\cdot) C_{2} x\right]+\sum_{j \in D_{i}} g_{\alpha_{n}-\alpha_{j}} *\left[R_{i}(\cdot) A_{j} x-\left(k * g_{i}\right)(\cdot) C_{2} A_{j} x\right]} \\
& +\sum_{j \in \mathbb{N}_{n-1} \backslash D_{i}}\left(g_{\alpha_{n}-\alpha_{j}} * R_{i}(\cdot) A_{j} x\right)(\cdot) \\
& \quad=\left\{\begin{array}{l}
\left(g_{\alpha_{n}-\alpha} * R_{i}(\cdot) A x\right)(\cdot), \quad m-1<i, \\
g_{\alpha_{n}-\alpha} *\left[R_{i}(\cdot) A x-\left(k * g_{i}\right)(\cdot) C_{2} A x\right](\cdot), \quad m-1 \geq i,
\end{array}\right.
\end{aligned}
$$

for any $x \in \bigcap_{0 \leq j \leq n-1} D\left(A_{j}\right)$ and $i \in \mathbb{N}_{m_{n}-1}^{0}$.

(iii) $k$-regularized $C$-resolvent propagation family for $(0.1)$, in short $k$-regularized $C$-propagation family for $(0.1)$, if $\left(\left(R_{0}(t)\right)_{t \in[0, \tau)}, \cdots,\left(R_{m_{n}-1}(t)\right)_{t \in[0, \tau)}\right)$ is a $k$ regularized $C$-uniqueness propagation family for (0.1), and if for every $t \in$ $[0, \tau), i \in \mathbb{N}_{m_{n}-1}^{0}$ and $j \in \mathbb{N}_{n-1}^{0}$, one has $R_{i}(t) A_{j} \subseteq A_{j} R_{i}(t), R_{i}(t) C=C R_{i}(t)$ and $C A_{j} \subseteq A_{j} C$.

As mentioned in the introductory part, the case in which the operator $A_{j}$ is a scalar multiple of the identity operator for all $j \in \mathbb{N}_{n-1}$ (cf. [21] for more details) is very specific. In the subsequent theorems, we shall prove certain relations between $\left(k, C_{1}, C_{2}\right)$-existence and uniqueness families $((k, C)$-resolvent families) and $k$-regularized $\left(C_{1}, C_{2}\right)$-existence and uniqueness propagation families ( $k$-regularized $C$-resolvent propagation families).

Theorem 2.1. (i) Let $C, C_{1} \in L(X)$, and let $C$ be injective. Suppose that $A_{j} \in$ $L(X)$ and $A_{j} A_{l}=A_{l} A_{j}$ for $1 \leq j, l \leq n-1$ and $A_{j} A \subseteq A A_{j}$ for $1 \leq j \leq n-1$. Suppose $(E(t))_{t \in[0, \tau)}$ is a $\left(k, C_{1}\right)$-existence family for $(0.1)$, and $(R(t))_{t \in[0, \tau)}$ is $a(k, C)$-resolvent family for (0.1). Put, for every $x \in X, t \in[0, \tau)$, and $i=m, \cdots, m_{n}-1$, 


$$
E_{i}(t) x:=\left(g_{i} * E\right)(t) x+\sum_{j \in D_{i}} A_{j}\left(g_{\alpha_{n}-\alpha_{j}+i} * E\right)(t) x,
$$

and, for every $x \in X, t \in[0, \tau)$, and $i=0, \cdots, m-1$,

$$
E_{i}(t) x:=\left(k * g_{i}\right)(t) C_{1} x-\sum_{j \in \mathbb{N}_{n-1} \backslash D_{i}} A_{j}\left(g_{\alpha_{n}-\alpha_{j}+i} * E\right)(t) x .
$$

Define also $R_{i}(t) x$ by replacing respectively $E(t)$ and $C_{1}$ with $R(t)$ and $C$ in the above formulae. Then $\left(\left(E_{0}(t)\right)_{t \in[0, \tau)}, \cdots,\left(E_{m_{n}-1}(t)\right)_{t \in[0, \tau)}\right)$ is a k-regularized $C_{1}$ existence propagation family for $(0.1)$ and $\left(\left(R_{0}(t)\right)_{t \in[0, \tau)}, \cdots,\left(R_{m_{n}-1}(t)\right)_{t \in[0, \tau)}\right)$ is a $k$-regularized $C$-resolvent propagation family for (0.1). Furthermore, (2.5) holds for $\left(\left(R_{0}(t)\right)_{t \in[0, \tau)}, \cdots,\left(R_{m_{n}-1}(t)\right)_{t \in[0, \tau)}\right)$, provided that $(2.2)$ holds for $(R(t))_{t \in[0, \tau)}$.

(ii) Let the following condition hold:

$$
c_{j} \in \mathbb{C} \text { and } A_{j}=c_{j} I \text { for all } j \in \mathbb{N}_{n-1} .
$$

Suppose $\left(\left(R_{0}(t)\right)_{t \in[0, \tau)}, \cdots,\left(R_{m_{n}-1}(t)\right)_{t \in[0, \tau)}\right)$ is a $k$-regularized $C_{1}$-existence propagation family for $(0.1)$, resp. $k$-regularized $C_{2}$-uniqueness propagation family for (0.1) (k-regularized $C$-resolvent propagation family for $(0.1)$ ), and $m=0$. Define, for every $t>0$,

$$
b(t):=\mathcal{L}^{-1}\left(\left(1+\sum_{j \in D_{0}} c_{j} \lambda^{\alpha_{j}-\alpha_{n}}\right)^{-1}-1\right)(t),
$$

and $R(t) x:=R_{0}(t) x+\left(b * R_{0}\right)(t) x, \quad t \in[0, \tau), x \in X$. Then $(R(t))_{t \in[0, \tau)}$ is a $\left(k, C_{1}\right)$-existence family for $(0.1)$, resp. $\left(k, C_{2}\right)$-uniqueness family for $(0.1)$ $((k, C)$-resolvent family for (0.1)). Furthermore, if $(2.5)$ holds for $\left(\left(R_{0}(t)\right)_{t \in[0, \tau)}, \cdots,\left(R_{m_{n}-1}(t)\right)_{t \in[0, \tau)}\right)$, then $(2.2)$ holds with $(R(t))_{t \in[0, \tau)}$ in place of $(E(t))_{t \in[0, \tau)}$.

Proof. We wil only prove the assertion (i) in the case of existence families. Suppose first $i \in \mathbb{N}_{m_{n}-1}^{0}$ and $m-1<i$. Then it is clear that $\left(E_{1, i}(t)\right)_{t \in[0, \tau)}$ is a strongly continuous operator family. Using the definition of $\left(E_{1, i}(t)\right)_{t \in[0, \tau)}$ and $(E(t))_{t \in[0, \tau)}$, as well as the equalities $A_{j} A_{l}=A_{l} A_{j}$ for $1 \leq j, l \leq n-1$ and $A_{j} A \subseteq A A_{j}$ for $1 \leq j \leq n-1$, we get that, for every $t \in[0, \tau)$ and $x \in X$,

$$
\begin{aligned}
& A\left(g_{\alpha_{n}-\alpha} * E_{1, i}\right)(t) x=g_{i} *\left[E_{1}(\cdot) x-k(\cdot) C_{1} x+\sum_{j=1}^{n-1} A_{j}\left(g_{\alpha_{n}-\alpha_{j}} * E_{1}\right)(\cdot) x\right](t) \\
& \quad+A\left(\sum_{j \in D_{i}} A_{j}\left(g_{\alpha_{n}-\alpha_{j}+i+\alpha_{n}-\alpha} * E_{1}\right)(t) x\right)
\end{aligned}
$$




$$
\begin{aligned}
= & g_{i} *\left[E_{1}(\cdot) x-k(\cdot) C_{1} x+\sum_{j=1}^{n-1} A_{j}\left(g_{\alpha_{n}-\alpha_{j}} * E_{1}\right)(\cdot) x\right](t) \\
& +\sum_{j \in D_{i}} A_{j}\left(g_{\alpha_{n}-\alpha_{j}+i} *\left[R_{1}(\cdot)-k(\cdot) C_{1}+\sum_{l=1}^{n-1} A_{l}\left(g_{\alpha_{n}-\alpha_{l}} * R_{1}\right)(\cdot)\right]\right)(t) x \\
= & {\left[R_{1, i}(\cdot) x-\left(k * g_{i}\right)(\cdot) C_{1} x\right](t)+\sum_{j \in \mathbb{N} \backslash D_{i}} A_{j}\left(g_{\alpha_{n}-\alpha_{j}} *\left[g_{i} * R_{1}\right]\right)(t) x } \\
& +\sum_{j \in D_{i}} A_{j}\left(g_{\alpha_{n}-\alpha_{j}+i} *\left[R_{1}(\cdot)-k(\cdot) C_{1}+\sum_{l=1}^{n-1} A_{l}\left(g_{\alpha_{n}-\alpha_{l}} * R_{1}\right)(\cdot)\right]\right)(t) x \\
= & {\left[R_{1, i}(\cdot) x-\left(k * g_{i}\right)(\cdot) C_{1} x\right](t) } \\
& +\sum_{j \in \mathbb{N} \backslash D_{i}} A_{j}\left(g_{\alpha_{n}-\alpha_{j}} *\left[R_{1, i}(\cdot)-\sum_{l \in D_{i}} A_{l}\left(g_{\alpha_{n}-\alpha_{j}+i} * R_{1}\right)(\cdot)\right]\right)(t) x \\
& +\sum_{j \in D_{i}}\left(g_{\alpha_{n}-\alpha_{j}} *\left[R_{1, i}(\cdot) x-\sum_{l \in D_{i}} A_{l}\left(g_{\alpha_{n}-\alpha_{j}+i} * R_{1}\right)(\cdot) x-\left(k * g_{i}\right)(\cdot) C_{1} x\right]\right. \\
& \left.+g_{\alpha_{n}-\alpha_{j}+i} * \sum_{l=1}^{n-1} A_{l}\left(g_{\alpha_{n}-\alpha_{l}} * R_{1}\right)(\cdot) x\right)(t) .
\end{aligned}
$$

Since, for every $t \in[0, \tau)$ and $x \in X$,

$$
\begin{aligned}
& -\sum_{j \in \mathbb{N} \backslash D_{i}} \sum_{l \in D_{i}} A_{j} A_{l}\left(g_{\alpha_{n}-\alpha_{l}+i+\alpha_{n}-\alpha_{j}} * R_{1}\right)(t) x-\sum_{j \in D_{i}} \sum_{l \in D_{i}} A_{j} A_{l}\left(g_{\alpha_{n}-\alpha_{l}+i+\alpha_{n}-\alpha_{j}} * R_{1}\right)(t) x \\
& =\sum_{l=1}^{n-1} \sum_{j \in D_{i}} A_{j} A_{l}\left(g_{\alpha_{n}-\alpha_{l}+i+\alpha_{n}-\alpha_{j}} * R_{1}\right)(t) x,
\end{aligned}
$$

(apply the substitution $(j, l) \hookrightarrow(l, j))$, the above implies $(2.5)$, with $\left(R_{i}(t)\right)_{t \in[0, \tau)}$ replaced by $\left(E_{1, i}(t)\right)_{t \in[0, \tau)}$. The proof in case $m-1 \geq i$ is similar and therefore omitted.

Let (2.7) hold. Then the relations between $k$-regularized $C$-resolvent propagation families and $(k, C)$-resolvent families are not clear in the case $m>0$. Here we recognize the following subcases:

(a) There exists $i \in \mathbb{N}_{m_{n}-1}^{0}$ such that $i>m-1$. Then the consideration is trivial provided that, for such an index $i$, one has $D_{i}=\emptyset$ (cf. Theorem 2.1); because of that, we shall assume in the further analysis of this subcase that $D_{i} \neq \emptyset$. 
(b) $m=m_{n}$ and, for every $i \in \mathbb{N}_{m_{n}-1}^{0}$, one has $\mathbb{N}_{n-1} \backslash D_{i}=\emptyset$. That is the worst case possible and here we have hat the function $u(t)=\sum_{k=0}^{m_{n}-1} u_{k} g_{k+1}(t), t \geq 0$ is a strong solution of $(0.1)$ since $\mathbf{D}_{t}^{\beta} g_{\gamma}(t) \equiv 0$, provided $\beta>\gamma-1>0$.

(c) $m=m_{n}$, and there exists $i \in \mathbb{N}_{m_{n}-1}^{0}$ such that $\mathbb{N}_{n-1} \backslash D_{i} \neq \emptyset$.

The proof of following theorem, which considers the cases (a) and (c) in more detail, is omitted for the sake of brevity.

Theorem 2.2. (i) Let $m>0$, and let $i \in \mathbb{N}_{m_{n}-1}^{0}$ satisfy $i>m-1$ and $D_{i} \neq \emptyset$. Suppose $l \in \mathbb{N}$ and there exists $j \in \mathbb{N}_{n-1} \backslash D_{i}$ such that $\alpha_{j}-\alpha_{n}+l>i$. Let $\left(\left(R_{0}(t)\right)_{t \in[0, \tau)}, \cdots,\left(R_{m_{n}-1}(t)\right)_{t \in[0, \tau)}\right)$ be a $k$-regularized $C_{1}$-existence propagation family for $(0.1)$, resp. $k$-regularized $C_{2}$-uniqueness propagation family for (0.1) (k-regularized $C$-resolvent propagation family for (0.1)). Suppose, additionally, that for every $x \in X$ and $p \in \circledast$, the mapping $t \mapsto R_{i}^{(l)}(t) x, t \in[0, \tau)$ is continuous with $R_{i}^{(j)}(0) x=0$ for all $j \in \mathbb{N}_{l-1}^{0}$, and that, for every $p \in \circledast$ and $t \in[0, \tau)$, there exist $c_{p, t}>0$ and $q_{p, t} \in \circledast$ such that $p\left(R_{i}^{(l)}(t) x\right) \leq c_{p, t} q_{p, t}(x)$, $x \in X$. Put, for every $t \in[0, \tau)$ and $x \in X$,

$$
R(t) x:=\left[\mathcal{L}^{-1}\left(\frac{1}{\lambda^{l-i}+\sum_{j \in D_{i}} c_{j} \lambda^{\alpha_{j}-\alpha_{n}-i+l}}\right) * R_{i}^{(l)}(\cdot)\right](t) x .
$$

Then $(R(t))_{t \in[0, \tau)}$ is a $\left(k, C_{1}\right)$-existence family for $(0.1)$, resp. $\left(k, C_{2}\right)$-uniqueness family for $(0.1)((k, C)$-resolvent propagation family for $(0.1))$. Furthermore, if (2.5) holds for $\left(\left(R_{0}(t)\right)_{t \in[0, \tau)}, \cdots,\left(R_{m_{n}-1}(t)\right)_{t \in[0, \tau)}\right)$, then (2.2) holds with $(R(t))_{t \in[0, \tau)}$ in place of $(E(t))_{t \in[0, \tau)}$.

(ii) Let $m=m_{n}$, and let $i \in \mathbb{N}_{m_{n}-1}^{0}$ be such that $\mathbb{N}_{n-1} \backslash D_{i} \neq \emptyset$. Suppose $\left(\left(R_{0}(t)\right)_{t \in[0, \tau)}, \cdots,\left(R_{m_{n}-1}(t)\right)_{t \in[0, \tau)}\right)$ is a $k$-regularized $C_{1}$-existence propagation family for $(0.1)$, resp. $k$-regularized $C_{2}$-uniqueness propagation family for (0.1) (k-regularized $C$-resolvent propagation family for $(0.1)$ ). Let $l \in \mathbb{N}$, let $c(t) \in C([0, \tau))$ satisfy $\tilde{c}(\lambda)=\tilde{k}(\lambda) \sum_{j \in \mathbb{N}_{n-1} \backslash D_{i}} c_{j} \lambda^{\alpha_{j}-\alpha_{n}}$, for $\lambda$ sufficiently large, and let there exist $j \in \mathbb{N}_{n-1} \backslash D_{i}$ such that $\alpha_{j}-\alpha_{n}+l>i$. Suppose, additionally, that for every $x \in X$ and $p \in \circledast$, the mapping $t \mapsto R_{i}^{(l)}(t) x, t \in[0, \tau)$ is continuous with $R_{i}^{(j)}(0) x=0$ for all $j \in \mathbb{N}_{l-1}^{0}$ and that, for every $p \in \circledast$ and $t \in[0, \tau)$, there exist $c_{p, t}>0$ and $q_{p, t} \in \circledast$ such that $p\left(R_{i}^{(l)}(t) x\right) \leq c_{p, t} q_{p, t}(x)$, $x \in X$. Put, for every $t \in[0, \tau)$ and $x \in X$,

$$
R(t) x:=c(t) C x-\left[\mathcal{L}^{-1}\left(\frac{1}{\sum_{j \in \mathbb{N}_{n-1} \backslash D_{i}} c_{j} \lambda^{\alpha_{j}-\alpha_{n}-i+l}}\right) * R_{i}^{(l)}(\cdot)\right](t) x .
$$

Then $(R(t))_{t \in[0, \tau)}$ is a $\left(k, C_{1}\right)$-existence family for $(0.1)$, resp. $\left(k, C_{2}\right)$-uniqueness family for $(0.1)((k, C)$-resolvent family for (0.1)). Furthermore, if (2.5) holds for $\left(\left(R_{0}(t)\right)_{t \in[0, \tau)}, \cdots,\left(R_{m_{n}-1}(t)\right)_{t \in[0, \tau)}\right)$, then $(2.2)$ holds with $(R(t))_{t \in[0, \tau)}$ in place of $(E(t))_{t \in[0, \tau)}$. 
Remark 2.1. Let (2.7) hold.

(i) Let $m=0$. Then it is checked at once that there exist $M \geq 1$ and $\omega \geq 0$ such that $\int_{0}^{t}|b(s)| d s \leq M e^{\omega t}, t \geq 0$, which implies that the properties of (q)exponential equicontinuity and local equicontinuity are stable under passing from $k$-regularized $C_{1}$-existence propagation families $\left(C_{2}\right.$-uniqueness propagation families, $C$-resolvent propagation families) to $\left(k, C_{1}\right)$-existence families $\left(\left(k, C_{2}\right)\right.$-uniqueness families, $(k, C)$-resolvent families). Suppose now that $\left(\left(R_{0}(t)\right)_{t \geq 0}, \cdots,\left(R_{m_{n}-1}(t)\right)_{t \geq 0}\right)$ is an exponentially equicontinuous, analytic $k$ regularized $C_{1}$-existence family $\left(C_{2}\right.$-uniqueness family, $C$-resolvent propagation family) of angle $\delta \in(0, \pi / 2]$. Owing to [18, Theorem 3.4(i)], we have that $(R(t))_{t \geq 0}$ is an exponentially equicontinuous, analytic $\left(k, C_{1}\right)$-existence family $\left(\left(k, C_{2}\right)\right.$-uniqueness family, $(k, C)$-resolvent family) of angle $\delta$.

(ii) Let $\left(\left(R_{0}(t)\right)_{t \in[0, \tau)}, \cdots,\left(R_{m_{n}-1}(t)\right)_{t \in[0, \tau)}\right)$ be a locally equicontinuous $k$-regularized $C$-resolvent propagation family for $(0.1)$, and let $m=0$. Then it is not difficult to see, with the help of Theorem 2.1-Theorem 2.2, that the following equality holds, for every $x \in D(A), i \in \mathbb{N}_{m_{n}-1}^{0}$ and $t \in[0, \tau)$,

$$
\begin{aligned}
R_{i}(t) x= & g_{i} *\left[R_{0}(\cdot) x+\left(b * R_{0}\right)(\cdot) x\right](t) \\
& +\sum_{j \in D_{i}} c_{j}\left[g_{\alpha_{n}-\alpha_{j}+i} *\left(R_{0}(\cdot) x+\left(b * R_{0}(\cdot) x\right)\right)\right](t) x
\end{aligned}
$$

furthermore, (2.9) holds for every $x \in X, i \in \mathbb{N}_{m_{n}-1}^{0}$ and $t \in[0, \tau)$, provided (2.5).

(iii) Consider the situation of [20, Example 5.1(b)]. Then there exists an exponentially equicontinuous, analytic $k_{1}$-regularized $I$-resolvent propagation family $\left(\left(R_{0}(t)\right)_{t \geq 0}, \cdots,\left(R_{m_{n}-1}(t)\right)_{t \geq 0}\right)$ for the corresponding problem $(0.1)$, with $k_{1}(t)$ being defined by $k_{1}(t)=\mathcal{L}^{-1}\left(\exp \left(-a_{1} \lambda^{b_{1}}\right)\right)(t), t \geq 0$ for certain positive real numbers $a_{1}>0$ and $b_{1} \in(0,1)$. Under some additional assumptions (very natural in the theory of convoluted operator families), we may apply Theorem 2.2 in the construction of an exponentially equicontinuous, analytic $\left(k_{1}, I\right)$ resolvent family for (0.1).

The following proposition is very similar to [20, Proposition 3.2] and [23, Proposition 2.7]. Because of that, we shall omit the proof.

Proposition 2.1. Suppose (2.7) holds, $\left(\left(R_{1,0}(t)\right)_{t \in[0, \tau)}, \cdots,\left(R_{1, m_{n}-1}(t)\right)_{t \in[0, \tau)}\right)$ is a $k$ regularized $C_{1}$-existence propagation family for $(0.1), \mathbb{N}_{n-1} \backslash D_{i} \neq \emptyset$ provided $m-1 \geq i$, and $\left(\left(R_{2,0}(t)\right)_{t \in[0, \tau)}, \cdots,\left(R_{2, m_{n}-1}(t)\right)_{t \in[0, \tau)}\right)$ is a locally equicontinuous $k$-regularized $C_{2}$-uniqueness propagation family for (0.1). Then, for every $i \in \mathbb{N}_{m_{n}-1}^{0}$, one has $C_{2} R_{1, i}(t)=R_{2, i}(t) C_{1}, t \in[0, \tau)$.

Before discussing some perturbation properties of (0.1), we would like to observe that Proposition 2.1 can be proved under slightly weakened assumptions (see e.g. the formulation of [20, Proposition 2.3]). Consider now the abstract Cauchy problem 


$$
\begin{aligned}
& \mathbf{D}_{t}^{\alpha_{n}} u(t)+\sum_{i=1}^{n-1}\left(A_{i}+B_{i}\right) \mathbf{D}_{t}^{\alpha_{i}} u(t)=(A+B) \mathbf{D}_{t}^{\alpha} u(t)+f(t), \quad t>0, \\
& u^{(k)}(0)=u_{k}, \quad k=0, \cdots,\left\lceil\alpha_{n}\right\rceil-1,
\end{aligned}
$$

where $B$ and $B_{1}, \cdots, B_{n-1}$ are closed linear operators on $E$. Put $B_{0}:=B$.

The following theorem is an important extension of [7, Theorem 6.2-Theorem 6.3].

Theorem 2.3. $\quad$ (i) Suppose $Y=X,(E(t))_{t \in[0, \tau)} \subseteq L(X)$ is a (local) $C_{1}$-existence family for $(0.1), D_{j} \in L(E)$ and $B_{j}=C_{1} D_{j}\left(j \in \mathbb{N}_{n-1}^{0}\right)$. Suppose that the following conditions hold:

(a) For every $p \in \circledast_{X}$ and for every $T \in(0, \tau)$, there exists $c_{p, T}>0$ such that $p\left(E^{\left(m_{n}-1\right)}(t) x\right) \leq c_{p, T} p(x), \quad x \in E, t \in[0, T]$.

(b) For every $p \in \circledast_{X}$, there exists $c_{p}>0$ such that $p\left(D_{j} x\right) \leq c_{p} p(x)$, $j \in \mathbb{N}_{n-1}^{0}, x \in E$.

(c) $\alpha_{n}-\alpha_{n-1} \geq 1$ and $\alpha_{n}-\alpha \geq 1$.

Then there exists a (local) $C_{1}$-existence propagation family $(R(t))_{t \in[0, \tau)}$ for (2.10). If $\tau=\infty$ and if, for every $p \in \circledast_{X}$, there exist $M \geq 1$ and $\omega \geq 0$ such that

$$
p\left(E^{\left(m_{n}-1\right)}(t) x\right) \leq M e^{\omega t} p(x), \quad t \geq 0, x \in E,
$$

then $(R(t))_{t \geq 0}$ is exponentially equicontinuous, and moreover, $(R(t))_{t \geq 0}$ also satisfies the condition (2.11), with possibly different numbers $M \geq 1$ and $\omega>0$.

(ii) Suppose $Y=X,(U(t))_{t \in[0, \tau)} \subseteq L(X)$ is a (local) $\left(1, C_{2}\right)$-uniqueness family for (0.1), $D_{j} \in L(E)$ and $B_{j}=D_{j} C_{2}\left(j \in \mathbb{N}_{n-1}^{0}\right)$ Suppose that (b)-(c) hold, and that (a) holds with $\left(E^{\left(m_{n}-1\right)}(t)\right)_{t \in[0, \tau)}$ replaced by $(U(t))_{t \in[0, \tau)}$ therein. Then there exists a (local) $\left(1, C_{2}\right)$-uniqueness family $(W(t))_{t \in[0, \tau)}$ for $(2.10)$. If $\tau=$ $\infty$ and if, for every $p \in \circledast_{X}$, there exist $M \geq 1$ and $\omega \geq 0$ such that $(2.11)$ holds, then $(W(t))_{t \geq 0}$ is exponentially equicontinuous, and moreover, $(W(t))_{t \geq 0}$ also satisfies the condition (2.11), with possibly different numbers $M \geq 1$ and $\omega>0$.

Proof. We will only outline the main details of proof. Put

$$
K_{0}(t) x:=-\left\{E^{\left(m_{n}-1\right)} *\left[\sum_{j=1}^{n-1} g_{\alpha_{n}-\alpha_{j}} D_{j}+g_{\alpha_{n}-\alpha} D\right]\right\}(t) x, \quad t \in[0, \tau), x \in X .
$$

Then the assumption (c) implies that, for every fixed $x \in X$, one has $K_{0}(\cdot) x \in$ $C^{1}([0, \tau): X)$ and

$$
K_{0}^{\prime}(t) x:=-\left\{E^{\left(m_{n}-1\right)} *\left[\sum_{j=1}^{n-1} g_{\alpha_{n}-\alpha_{j}-1} D_{j}+g_{\alpha_{n}-\alpha-1} D\right]\right\}(t) x, \quad t \in[0, \tau) .
$$


Denote by $f^{*, k}(t)$ the $k$ th convolution power of a function $f(t)$. By assumptions (a)(b), we have that, for every $t \in[0, \tau)$ and $x \in X$, the series

$$
L(t) x:=-\left[K_{0}(t) x+\left(K_{0} * K_{0}^{\prime}\right)(t) x+\cdots+\left(K_{0} * K_{0}^{\prime, * k}\right)(t) x+\cdots\right]
$$

converges, uniformly on compacts of $[0, \tau)$. Furthermore, the operator family $(L(t))_{t \in[0, \tau)} \subseteq L(X)$ is strongly continuous and, for every $x \in X$, the unique solution of the following integral equation:

$$
R_{m_{n}-1}(t) x=E^{\left(m_{n}-1\right)}(t) x+\int_{0}^{t} K_{0}^{\prime}(t-s) R_{m_{n}-1}(s) x d s, \quad t \in[0, \tau),
$$

is given by

$$
R_{m_{n}-1}(t) x=E^{\left(m_{n}-1\right)}(t) x+\int_{0}^{t} L^{\prime}(t-s) E^{\left(m_{n}-1\right)}(s) x d s, \quad t \in[0, \tau) ;
$$

cf. also [30, Theorem 0.5, Corollary 0.3]. It is not difficult to prove that $\left(R_{m_{n}-1}(t)\right)_{t \in[0, \tau)}$ is a strongly continuous operator family in $L(X)$. Define now $R(t) x:=\left(g_{m_{n}-1} *\right.$ $\left.R_{m_{n}-1}\right)(t) x, t \in[0, \tau), x \in X$. Applying the functional equation for $(E(t))_{t \in[0, \tau)}$ twice, it is checked at once that $(R(t))_{t \in[0, \tau)}$ satisfies

$$
\begin{aligned}
& R^{\left(m_{n}-1\right)}(t) x+\sum_{j=1}^{n-1}\left(A_{j}+B_{j}\right)\left(g_{\alpha_{n}-\alpha_{j}} * R^{\left(m_{n}-1\right)}\right)(t) x \\
& \quad-(A+B)\left(g_{\alpha_{n}-\alpha} * R^{\left(m_{n}-1\right)}\right)(t) x=C_{1} x, \quad t \in[0, \tau), x \in X,
\end{aligned}
$$

if we prove that, for every $t \in[0, \tau)$ and $x \in X$,

$$
\begin{aligned}
& \left(d K_{0} * R_{m_{n}-1}\right)(t) x+\left(E^{\left(m_{n}-1\right)} * \sum_{j=0}^{n-1} g_{\alpha_{n}-\alpha_{j}-1}(\cdot) D_{j} * R_{m_{n}-1}\right)(t) x \\
& +\left(C_{1} * \sum_{j=0}^{n-1} g_{\alpha_{n}-\alpha_{j}-1}(\cdot) D_{j} * R_{m_{n}-1}\right)(t) x \\
& \quad+\sum_{j=1}^{n-1} B_{j}\left(g_{\alpha_{n}-\alpha_{j}} * R_{m_{n}-1}\right)(t) x-B\left(g_{\alpha_{n}-\alpha} * R_{m_{n}-1}\right)(t) x=0 .
\end{aligned}
$$

But, the last equality is an immediate consequence of (2.12). In the case that $\left(E^{\left(m_{n}-1\right)}(t)\right)_{t \geq 0}$ satisfies $(2.11)$, then it is not difficult to see, with the help of (2.12)(2.13), that $(R(t))_{t \in[0, \tau)}$ also satisfies the same condition, with possibly different numbers $M \geq 1$ and $\omega>0$. The proof of (ii) is quite similar. Define, for every $t \in[0, \tau)$ and $x \in X$,

$$
Q_{0}(t) x:=-\left\{\left[\sum_{j=1}^{n-1} g_{\alpha_{n}-\alpha_{j}} D_{j}+g_{\alpha_{n}-\alpha} D\right] * U\right\}(t) x,
$$


and $Z(t) x:=-\left[Q_{0}(t) x+\left(Q_{0} * Q_{0}^{\prime}\right)(t) x+\cdots+\left(Q_{0} * Q_{0}^{\prime * *}\right)(t) x+\cdots\right]$.

The resulting $\left(1, C_{2}\right)$-uniqueness family $(W(t))_{t \in[0, \tau)}$ is the unique solution of the following integral equation:

$$
W(t) x=U(t) x+\int_{0}^{t} W(t-s) Q^{\prime}(s) x d s, \quad t \in[0, \tau), x \in X,
$$

which is given by $W(t) x=U(t) x+\int_{0}^{t} U(t-s) Z^{\prime}(s) x d s, t \in[0, \tau), x \in X$. The proof is completed through a routine argument.

The analysis of persistence of differential and analytical properties under perturbations described in Theorem 2.3 will appear elsewhere.

Remark 2.2. (i) It is worth noting that the proof of the preceding theorem is a slight modification of the corresponding proofs of [30, Theorem 6.1] and [24, Theorem 2.12], established for abstract Volterra equations of non-scalar type. Using the method given in the proofs of aforementioned theorems, one can similarly clarify some results on time-dependent perturbations of (0.1).

(ii) It is not clear how one can prove an analogue of Theorem 2.3(ii) in the case of a (local) $C_{2}$-uniqueness family for (0.1).

Example 2.1. Suppose $1 \leq p \leq \infty, X:=L^{p}(\mathbb{R}), a \in \mathbb{R}, r>0, \vartheta(\cdot) \in W^{1, \infty}(\mathbb{R})$, $1 / 2<\gamma \leq 1, T>0, \chi \in C([0, T]: X)$, and $\frac{d}{d t}\left(g_{2 \gamma-1} * \frac{d}{d x} \chi(t, \cdot)\right) \in C([0, T]: X)$. Put $A_{1}:=a d / d x$ and $A u:=r \Delta u-\vartheta(\cdot) u$ with maximal distributional domain. In [20, Example 5.3], we have recently considered the following fractional analogue of the damped Klein-Gordon equation:

$$
\mathbf{D}_{t}^{2 \gamma} u(t, x)+a \frac{\partial}{\partial x} \mathbf{D}_{t}^{\gamma} u(t, x)-r \Delta_{x} u(t, x)+\vartheta(x) u(t, x)=\chi(t, x),
$$

where $t>0, x \in \mathbb{R}$, and $u(0, x)=\phi(x), u_{t}(0, x)=\psi(x), x \in \mathbb{R}$, continuing the analysis of T.-J. Xiao and J. Liang [33, Example 4.1], in which it has been assumed that $\gamma=1$. Since $\alpha_{2}-\alpha_{1}=\gamma<1$ for $1 / 2<\gamma<1$, it is clear that Theorem 2.3 cannot be applied in this case, unfortunately. If $\gamma=1$, then Theorem 2.3 is applicable and we shall consider, as an illustration, the following perturbed problem of (2.14):

$$
\begin{aligned}
& \frac{\partial^{2} u(t, x)}{\partial t^{2}}+a \frac{\partial^{2} u(t, x)}{\partial x \partial t}+\int_{-\infty}^{+\infty} f(x-y) \frac{\partial u(s, y)}{\partial s} d y \\
& -r \frac{\partial^{2} u(t, x)}{\partial x^{2}}+\vartheta(x) u(t, x)+\int_{-\infty}^{+\infty} g(x-y) u(t, y) d y=0, \quad t>0, x \in \mathbb{R},
\end{aligned}
$$

where $u(0, x)=\phi(x), u_{t}(0, x)=\psi(x), \quad x \in \mathbb{R}$, with $f, g \in W^{1,1}(\mathbb{R})$. Making use of Theorem 2.3 and the corresponding result with $f=g=0$, we get that for each 
$\mu_{0} \in \rho\left(A_{1}\right)$ there exists a global exponentially bounded $\left(\mu_{0}-A_{1}\right)^{-1}$-existence family for (2.15). By [20, Theorem 3.7], it readily follows that, for every $\phi \in W^{3, p}(\mathbb{R})$ and $\psi \in W^{3, p}(\mathbb{R})$, there exists a strong solution $u(t, x)$ of the problem $(2.15)$ as well as that there exist $M \geq 1$ and $\omega \geq 0$ such that the following estimate holds for each $t \geq 0$ : $\|u(t, x)\|_{L^{p}(\mathbb{R})} \leq M e^{\omega t}\left[\|\phi\|_{W^{1, p}(\mathbb{R})}+\|\psi\|_{W^{1, p}(\mathbb{R})}\right]$. To prove the uniqueness of solutions to $(2.15)$, notice that there exist a number $\omega \geq 0$ and a strongly continuous operator family $(U(t))_{t \geq 0} \subseteq L\left(L^{p}(\mathbb{R})\right)$ such that, for every $h \in W^{2, p}(\mathbb{R}), \int_{0}^{\infty} e^{-\lambda t} U(t) h d t=$ $\left(\lambda^{2}+\lambda A_{1}+A_{0}\right)^{-1} h, \quad \Re \lambda>\omega ;$ cf. [33, Example 4.1]. Let $B_{0} \in L\left(L^{p}(\mathbb{R})\right)$ and $B_{1} \in L\left(L^{p}(\mathbb{R})\right)$ be defined by $B_{0} h=f * h$ and $B_{0} h=g * h$ for any $h \in L^{p}(\mathbb{R})$. Suppose, for the time being, that $\|f\|_{L^{1}(\mathbb{R})}$ is a sufficiently small positive real number. Making use of the complex characterization theorem for the Laplace transform and [31, Theorem 1.1.11], in this case we obtain that there exist a sufficiently large number $\omega^{\prime}>\omega$ and a strongly continuous operator family $\left(U_{1}(t)\right)_{t \geq 0} \subseteq L\left(L^{p}(\mathbb{R})\right)$ such that the operator $\left(\lambda^{2}+\lambda\left(A_{1}+B_{1}\right)+\left(A_{0}+B_{0}\right)\right)^{-1}$ exists in $L\left(L^{p}(\mathbb{R})\right)$ for any $\lambda \in \mathbb{C}$ with $\Re \lambda>\omega^{\prime}$, and that

$$
\begin{aligned}
\lambda^{-2} & \left(\lambda^{2}+\lambda\left(A_{1}+B_{1}\right)+\left(A_{0}+B_{0}\right)\right)^{-1} h \\
& =\lambda^{-2}\left(I+\left(\lambda^{2}+\lambda A_{1}+A_{0}\right)^{-1}\left(\lambda B_{1}+B_{0}\right)\right)^{-1}\left(\lambda^{2}+\lambda A_{1}+A_{0}\right)^{-1} h \\
& =\int_{0}^{\infty} e^{-\lambda t} U_{1}(t) h d t, \quad \Re \lambda>\omega^{\prime}, h \in L^{p}(\mathbb{R}) .
\end{aligned}
$$

Therefore, if $\|f\|_{L^{1}(\mathbb{R})}$ is sufficiently small, then we may apply [20, Theorem 3.5(iii)] in order to see that there exists a global exponentially bounded $g_{3}$-regularized $I$ uniqueness family for the perturbed problem (2.10). The final conclusion now follows from [20, Theorem 3.4(ii)] and the fact that, for every (sufficiently small) $c>0$, the function $v(t, x):=u(c t, x), t \geq 0, x \in \mathbb{R}$ satisfies the equation

$$
\begin{aligned}
& \frac{\partial^{2} v(t, x)}{\partial t^{2}}+a c \frac{\partial^{2} v(t, x)}{\partial x \partial t}+\int_{-\infty}^{+\infty} c f(x-y) \frac{\partial v(s, y)}{\partial s} d y \\
& -r c^{2} \frac{\partial^{2} u(t, x)}{\partial x^{2}}+c^{2} \vartheta(x) v(t, x)+\int_{-\infty}^{+\infty} c^{2} g(x-y) v(t, y) d y=0, \quad t>0, x \in \mathbb{R}
\end{aligned}
$$

where $v(0, x)=0, v_{t}(0, x)=0, x \in \mathbb{R}$, provided that $u(t, x)$ satisfies $(2.15)$ with $\phi=\psi=0$. Finally, we would like to mention that the slightly better results on the $C$-wellposedness of equation (2.15) can be obtained in the case that the function 
$\vartheta(\cdot)$ is a positive constant (cf. [15]), and that Theorem 2.3 can be also applied to differential operators considered in [23, Example 2.11-Example 2.12].

\section{Existence And Uniqueness of Solutions to (0.1)}

We start this section by stating the following existence type theorem.

Theorem 3.1. Suppose $A, A_{1}, \cdots, A_{n-1}$ are closed linear operators on $X, \omega>0$, $L(X) \ni C$ is injective and $u_{0}, \cdots, u_{m_{n}-1} \in X$. Set $P_{\lambda}:=\lambda^{\alpha_{n}-\alpha}+\sum_{j=1}^{n-1} \lambda^{\alpha_{j}-\alpha} A_{j}-A$, $\lambda \in \mathbb{C} \backslash\{0\}$. Let the following conditions hold:

(i) The operator $P_{\lambda}$ is injective for $\lambda>\omega$ and $D\left(P_{\lambda}^{-1} C\right)=X, \lambda>\omega$.

(ii) If $0 \leq j \leq n-1,0 \leq k \leq m_{n}-1, m-1<k, 1 \leq l \leq n-1, m_{l}-1 \geq k$ and $\lambda>\omega$, then $C u_{k} \in \bar{D}\left(P_{\lambda}^{-1} A_{l}\right)$,

$$
\begin{aligned}
& A_{j}\left\{\lambda^{\alpha_{j}}\left[\lambda^{\alpha_{n}-\alpha-k-1} P_{\lambda}^{-1} C u_{k}+\sum_{l \in D_{k}} \lambda^{\alpha_{l}-\alpha-k-1} P_{\lambda}^{-1} A_{l} C u_{k}\right]\right. \\
& \left.\quad-\sum_{l=0}^{m_{j}-1} \delta_{k l} \lambda^{\alpha_{j}-1-l} C u_{k}\right\} \in L T-X
\end{aligned}
$$

and

$$
\lambda^{\alpha_{n}}\left[\lambda^{\alpha_{n}-\alpha-k-1} P_{\lambda}^{-1} C u_{k}+\sum_{l \in D_{k}} \lambda^{\alpha_{l}-\alpha-k-1} P_{\lambda}^{-1} A_{l} C u_{k}\right]-\lambda^{\alpha_{n}-1-k} C u_{k} \in L T-X .
$$

(iii) If $0 \leq j \leq n-1,0 \leq k \leq m_{n}-1, m-1 \geq k, \mathbb{N}_{n-1} \backslash D_{k} \neq \emptyset, s \in \mathbb{N}_{n-1} \backslash D_{k}$ and $\bar{\lambda}>\omega$, then $C u_{k} \in \bar{D}\left(A_{s}\right), \sum_{l \in \mathbb{N}_{n-1} \backslash D_{k}} \lambda^{\alpha_{l}-\alpha-k-1} A_{l} C u_{k} \in D\left(P_{\lambda}^{-1}\right)$,

$$
\begin{aligned}
A_{j} & \left\{\lambda^{\alpha_{j}}\left[\lambda^{-k-1} C u_{k}-P_{\lambda}^{-1} \sum_{l \in \mathbb{N}_{n-1} \backslash D_{k}} \lambda^{\alpha_{l}-\alpha-k-1} A_{l} C u_{k}\right]\right. \\
& \left.-\sum_{l=0}^{m_{j}-1} \delta_{k l} \lambda^{\alpha_{j}-1-l} C u_{k}\right\} \in L T-X
\end{aligned}
$$

and

$$
\lambda^{\alpha_{n}}\left[\lambda^{-k-1} C u_{k}-P_{\lambda}^{-1} \sum_{l \in \mathbb{N}_{n-1} \backslash D_{k}} \lambda^{\alpha_{l}-\alpha-k-1} A_{l} C u_{k}\right]-\lambda^{\alpha_{n}-1-k} C u_{k} \in L T-X .
$$

Then the abstract Cauchy problem (0.1) has a strong solution, with $u_{k}$ replaced by $C u_{k}\left(0 \leq k \leq m_{n}-1\right)$.

Proof. Suppose, for the time being, $0 \leq k \leq m_{n}-1$ and $m-1<k$. Denote by $F_{k, n}:(\omega, \infty) \rightarrow X$ the function satisfying that, for every $p \in \circledast$, there exists $M_{p}>0$ such that $p\left(F_{k, n}(t)\right) \leq M_{p} e^{\omega t}, t \geq 0$, and 


$$
\begin{aligned}
\int_{0}^{\infty} e^{-\lambda t} F_{k, n}(t) d t= & \lambda^{\alpha_{n}}\left[\lambda^{\alpha_{n}-\alpha-k-1} P_{\lambda}^{-1} C u_{k}\right. \\
& \left.+\sum_{l \in D_{k}} \lambda^{\alpha_{l}-\alpha-k-1} P_{\lambda}^{-1} A_{l} C u_{k}\right]-\lambda^{\alpha_{n}-1-k} C u_{k}
\end{aligned}
$$

Then it is straightforward to see that there exists a function $u_{k}:(\omega, \infty) \rightarrow X$ such that, for every $p \in \circledast$, there exists $M_{p}^{\prime}>0$ satisfying $p\left(u_{k}(t)\right) \leq M_{p}^{\prime} e^{\omega t}, t \geq 0$, and

$$
\int_{0}^{\infty} e^{-\lambda t} u_{k}(t) d t=\lambda^{\alpha_{n}-\alpha-k-1} P_{\lambda}^{-1} C u_{k}+\sum_{l \in D_{k}} \lambda^{\alpha_{l}-\alpha-k-1} P_{\lambda}^{-1} A_{l} C u_{k}, \quad \lambda>\omega .
$$

The Laplace transform can be used to prove that:

$$
\left(g_{m_{n}} * F_{k, n}\right)(t)=\left[g_{m_{n}-\alpha_{n}} *\left(u_{k}(\cdot)-g_{k+1}(\cdot) C u_{k}\right)\right](t), \quad t \geq 0 .
$$

This implies $\left(g_{\alpha_{n}} * F_{k, n}\right)(t)=u_{k}(t)-g_{k+1}(t) C u_{k}, t \geq 0, u \in C^{m_{n}-1}([0, \infty): X)$ and $u_{k}^{(j)}(0)=\delta_{k j} C u_{k}$ for $0 \leq j \leq m_{n}-1$. Due to (3.5), we have $\mathbf{D}_{t}^{\alpha_{n}} u_{k}(t)=F_{k, n}(t), t \geq 0$. It is not difficult to show that, for every $j \in \mathbb{N}_{n-1}^{0}, \mathbf{D}_{t}^{\alpha_{j}} u_{k}$ is defined as well as that

$$
\int_{0}^{\infty} e^{-\lambda t} \mathbf{D}_{t}^{\alpha_{j}} u_{k}(t) d t=\lambda^{\alpha_{j}} \int_{0}^{\infty} e^{-\lambda t} u_{k}(t) d t-\sum_{l=0}^{m_{j}-1} u_{k}^{(l)}(0) \lambda^{\alpha_{j}-1-l}, \quad \lambda>\omega .
$$

Notice now that the condition (3.1) in combination with [31, Theorem 1.1.10] implies that the mapping $t \mapsto A_{j} \mathbf{D}_{t}^{\alpha_{j}} u_{k}(t), t \geq 0$ is well defined, continuous as well as that

$$
\int_{0}^{\infty} e^{-\lambda t} A_{j} \mathbf{D}_{t}^{\alpha_{j}} u_{k}(t) d t=A_{j}\left[\lambda^{\alpha_{j}} \int_{0}^{\infty} e^{-\lambda t} u_{k}(t) d t-\sum_{l=0}^{m_{j}-1} u_{k}^{(l)}(0) \lambda^{\alpha_{j}-1-l}\right], \lambda>\omega .
$$

Having in mind (3.4), (3.6) and the definition of $P_{\lambda}$, a simple calculation yields that:

$$
\int_{0}^{\infty} e^{-\lambda t}\left[\mathbf{D}_{t}^{\alpha_{n}} u_{k}(t)+A_{n-1} \mathbf{D}_{t}^{\alpha_{n-1}} u_{k}(t)+\cdots+A_{1} \mathbf{D}_{t}^{\alpha_{1}} u_{k}(t)-A \mathbf{D}_{t}^{\alpha} u_{k}(t)\right] d t=0,
$$

which implies that $u_{k}(\cdot)$ is a strong solution of the problem $(0.1)$ with $u_{k}^{(j)}(0)=\delta_{k j} C u_{k}$. Suppose now $0 \leq k \leq m_{n}-1$ and $m-1 \geq k$. Then one can similarly prove, with the help of conditions (3.2)-(3.3), that the function $t \mapsto u_{k}(t):=\mathcal{L}^{-1}\left(\lambda^{-k-1} C u_{k}-\right.$ $\left.P_{\lambda}^{-1} \sum_{l \in \mathbb{N}_{n-1} \backslash D_{k}} \lambda^{\alpha_{l}-\alpha-l-1} A_{l} C u_{k}\right)(t), t \geq 0$, is a strong solution of the problem $(0.1)$ with $u_{k}^{(j)}(0)=\delta_{k j} C u_{k}$. Define $u(t):=\sum_{k=0}^{m_{n}-1} u_{k}(t), t \geq 0$. Then it is clear that $u(\cdot)$ is a strong solution of the abstract Cauchy problem (0.1). 
Remark 3.1. (i) Let $0 \leq k \leq m_{n}-1$ and $m-1<k$. Then Theorem 3.1 continues to hold if we replace the term $\lambda^{\alpha_{n}-\alpha-k-1} P_{\lambda}^{-1} C u_{k}+\sum_{l \in D_{k}} \lambda^{\alpha_{l}-\alpha-k-1} P_{\lambda}^{-1} A_{l} C u_{k}$ i.e., the Laplace transform of $u_{k}(t)$, in (3.1) by

$$
\lambda^{-k-1} C u_{k}-\sum_{l \in \mathbb{N}_{n-1} \backslash D_{k}} \lambda^{\alpha_{l}-\alpha-k-1} P_{\lambda}^{-1} A_{l} C u_{k}+\lambda^{-k-1} P_{\lambda}^{-1} A C u_{k}
$$

in this case, one has to assume that $C u_{k} \in D\left(P_{\lambda}^{-1} A_{l}\right)$, provided $0 \leq l \leq n-1$, $k>m_{l}-1$ and $\lambda>\omega$. Notice also that a similar modification can be made in the case $0 \leq k \leq m_{n}-1$ and $m-1 \geq k$. As a matter of fact, one can replace the term $\lambda^{-k-1} C u_{k}-P_{\lambda}^{-1} \sum_{l \in \mathbb{N}_{n-1} \backslash D_{k}} \lambda^{\alpha_{l}-\alpha-k-1} A_{l} C u_{k}$ i.e., the Laplace transform of $u_{k}(t)$, in $(3.2)-(3.3)$ by

$$
\lambda^{\alpha_{n}-\alpha-k-1} P_{\lambda}^{-1} C u_{k}+\sum_{l \in D_{k}} \lambda^{\alpha_{l}-\alpha-k-1} P_{\lambda}^{-1} A_{l} C u_{k}-\lambda^{-k-1} P_{\lambda}^{-1} A C u_{k} ;
$$

in this case, one has to assume that $C u_{k} \in D\left(P_{\lambda}^{-1} A_{l}\right)$, provided $0 \leq l \leq n-1$, $m_{l}-1 \geq k$ and $\lambda>\omega$.

(ii) Consider now the situation of the abstract Cauchy problem $\left(\mathrm{ACP}_{n}\right)$, i.e., suppose that $\alpha_{j}=j, j \in \mathbb{N}_{n}^{0}$. Keeping in mind the proof of [31, Lemma 2.2.1, pp. 54-55], it readily follows that the condition:

$$
\lambda^{j} A_{j} P_{\lambda}^{-1} C u_{n-1}, \quad \lambda^{j} A_{j} \sum_{i=0}^{k} \lambda^{i-k-1} P_{\lambda}^{-1} A_{i} C u_{k} \in L T-X,
$$

for any $k \in \mathbb{N}_{n-2}^{0}$ and $j \in \mathbb{N}_{n-1}^{0}$, implies (3.1). Therefore, Theorem 3.1 can be viewed as a generalization of the above-mentioned result.

Now we shall state and prove the Ljubich uniqueness theorem for abstract timefractional equations of the form (0.1).

Theorem 3.2. Let $\lambda>0$, let $L(X) \ni C$ be injective, and let $D\left(P_{n \lambda}^{-1} C\right)=X, n \in \mathbb{N}$. Suppose, additionally, that for every positive real number $\sigma>0$ and for every null sequence $\left(x_{n}\right)_{n \in \mathbb{N}}$ in $X$, one has:

$$
\lim _{n \rightarrow \infty} e^{-n \lambda \sigma} P_{n \lambda}^{-1} C x_{n}=0 .
$$

Then, for every $u_{0}, \cdots, u_{m_{n}-1} \in X$, the abstract Cauchy problem (0.1) has at most one strong (integral) solution.

Proof. Clearly, it suffices to show the uniqueness of integral solutions of the abstract Cauchy problem $(0.1)$ with $u^{(k)}(0)=u_{k}=0, k \in \mathbb{N}_{m_{n}-1}^{0}$. Let $u(t)$ be such a solution. Then, for every $n \in \mathbb{N}$ and $t \geq 0$, one has: 


$$
\begin{aligned}
P_{n \lambda} \int_{0}^{t} e^{n \lambda(t-s)} C u(s) d s & \\
= & (n \lambda)^{\alpha_{n}-\alpha} \int_{0}^{t} e^{n \lambda(t-s)}\left[\left(g_{\alpha_{n}-\alpha} * C A u\right)(s)-\sum_{j=1}^{n-1}\left(g_{\alpha_{n}-\alpha_{j}} * C A_{j} u\right)(s)\right] d s \\
& +\sum_{j=1}^{n-1}(n \lambda)^{\alpha_{j}-\alpha} C A_{j} \int_{0}^{t} e^{n \lambda(t-s)} u(s) d s-C A \int_{0}^{t} e^{n \lambda(t-s)} u(s) d s \\
= & {\left[(n \lambda)^{\alpha_{n}-\alpha} \int_{0}^{t} e^{n \lambda(t-s)}\left(g_{\alpha_{n}-\alpha} * C A u\right)(s) d s-C A \int_{0}^{t} e^{n \lambda(t-s)} u(s) d s\right] } \\
& +\sum_{j=1}^{n-1}(n \lambda)^{\alpha_{j}-\alpha} C A_{j} \int_{0}^{t} e^{n \lambda(t-s)} u(s) d s \\
& -(n \lambda)^{\alpha_{n}-\alpha} \int_{0}^{t} e^{n \lambda(t-s)}\left(g_{\alpha_{n}-\alpha_{j}} * C A_{j} u\right)(s) d s .
\end{aligned}
$$

Keeping in mind [31, Lemma 1.5.5, p. 23] and its proof, we obtain that there exist numbers $M_{0}, \cdots, M_{n-1} \geq 1$ and $k_{0}, \cdots, k_{n-1} \in \mathbb{N}$ such that, for every $p \in \circledast, t \geq 0$, $n \in \mathbb{N}$ and $j \in \mathbb{N}_{n-1}^{0}$,

$$
\begin{aligned}
& p\left((n \lambda)^{\alpha_{n}-\alpha} \int_{0}^{t} e^{n \lambda(t-s)}\left(g_{\alpha_{n}-\alpha} * A u\right)(s) d s-A \int_{0}^{t} e^{n \lambda(t-s)} u(s) d s\right) \\
& \quad=p\left((n \lambda)^{\alpha_{n}-\alpha} \int_{0}^{t}\left(\int_{0}^{\infty} \frac{(s n \lambda+\varsigma)^{\alpha_{n}-\alpha-1}}{\Gamma\left(\alpha_{n}-\alpha\right)} e^{-\varsigma} d \varsigma\right) A u(t-s) d s\right) \\
& \leq M_{0}(1+n+|\lambda|)^{k_{0}} \int_{0}^{t} p(A u(s)) d s,
\end{aligned}
$$

and

$$
p\left((n \lambda)^{\alpha_{j}-\alpha} A_{j} \int_{0}^{t} e^{n \lambda(t-s)} u(s) d s-(n \lambda)^{\alpha_{n}-\alpha} \int_{0}^{t} e^{n \lambda(t-s)}\left(g_{\alpha_{n}-\alpha_{j}} * A_{j} u\right)(s) d s\right)
$$




$$
\begin{aligned}
& =p\left((n \lambda)^{\alpha_{n}-\alpha} \int_{0}^{t}\left[\int_{0}^{s} e^{n \lambda(s-r)} g_{\alpha_{n}-\alpha_{j}}(r) d r-e^{n \lambda s}(n \lambda)^{\alpha_{j}-\alpha_{n}}\right] A_{j} u(t-s) d s\right) \\
& \leq M_{j}(1+n+|\lambda|)^{k_{j}} \int_{0}^{t} p\left(A_{j} u(s)\right) d s .
\end{aligned}
$$

Using (3.8)-(3.12), it is not difficult to see that, for every $\sigma>0$ and $t \geq 0$, we have $\lim _{n \rightarrow \infty} e^{-n \lambda \sigma} \int_{0}^{t} e^{n \lambda(t-s)} C u(s) d s=0$. Since $\lim _{n \rightarrow \infty} \int_{t-\sigma}^{t} e^{n \lambda(t-s-\sigma)} C u(s) d s=0$ for $0 \leq \sigma \leq t$, we obtain that $\lim _{n \rightarrow \infty} \int_{0}^{t-\sigma} e^{n \lambda(t-s-\sigma)} C u(s) d s=0$. By [16, Lemma 3.5(iii)], one gets $C u(t)=0, t \geq 0$, which completes the proof by the injectivity of C.

If $\alpha_{n}-\alpha_{j} \in \mathbb{N}, j \in \mathbb{N}_{n-1}^{0}$, then the formulae (3.9) and (3.11) imply that it suffices to suppose (instead of a slightly stronger condition (3.7)) that, for every $\sigma>0$ and $x \in X$, one has $\lim _{n \rightarrow \infty} e^{-n \lambda \sigma} P_{n \lambda}^{-1} C x=0$; keeping this observation in mind, it readily follows that Theorem 3.2 provides a generalization of [14, Theorem 2.3.23] and [31, Lemma 2.3.1, pp. 67-68]. Notice also that the analysis given in [18, Remark 2.2] enables one to see that Theorem 3.2 provides a proper generalization of $[16$, Theorem $3.6]$ in barreled spaces.

Acknowledgment: The first and third author are supported by the NSFC of China (Grant No. 10971146). The second author is supported by Grant No. 174024 of Ministry of Science and Technological Development, Republic of Serbia.

\section{REFERENCES}

[1] W. Arendt, C. J. K. Batty, M. Hieber, F. Neubrander, Vector-valued Laplace Transforms and Cauchy Problems, Monographs in Mathematics, Vol. 96, Birkhäuser Verlag, Basel, 2001.

[2] E. Bajlekova, Fractional Evolution Equations in Banach Spaces, Ph.D. Dissertation, Eindhoven University of Technology, 2001.

[3] Yu. V. Bogacheva, Resolution's Problems of Initial Problems for Abstract Differential Equations with Fractional Derivatives. Ph.D. Dissertation, Belgorod, 2006.

[4] C. Chen, M. Li, On fractional resolvent operator functions, Semigroup Forum 80 (2010), 121142.

[5] C. Chen, M. Kostić, M. Li, Complex powers of almost $C$-sectorial operators, Contemp. Anal. Appl. Math., 2 (2014), 1-77.

[6] R. deLaubenfels, Existence Families, Functional Calculi and Evolution Equations, Lecture Notes in Mathematics, Vol. 1570, Springer, New York, 1994.

[7] R. deLaubenfels, Existence and uniqueness families for the abstract Cauchy problem, J. Lond. Math. Soc. 2(44) (1991), 310-338.

[8] K. Diethelm, The Analysis of Fractional Differential Equations, Springer-Verlag, Berlin, 2010.

[9] A. V. Glushak, A Cauchy-type problem for an abstract differential equation with fractional derivatives, Math. Notes 77 (2005), 26-38.

[10] A. V. Glushak, On the properties of a Cauchy-type problem for an abstract differential equation with fractional derivatives, Math. Notes 82 (2007), 596-607. 
[11] H. Jiang, F. Liu, I. Turner, K. Burrage, Analytical solutions for the multi-term time-space Caputo-Riesz fractional advection-diffusion equations on a finite domain, J. Math. Anal. Appl. 389 (2012), 1117-1127.

[12] V. Keyantuo, C. Lizama, A characterization of periodic solutions for time-fractional differential equations in UMD spaces and applications, Math. Nachr. 284 (2011), 494-506.

[13] V. Kiryakova, Generalized Fractional Calculus and Applications, Longman Scientific \& Technical, Harlow, 1994, copublished in the United States with John Wiley \& Sons, Inc., New York.

[14] M. Kostić, Generalized Semigroups and Cosine Functions, Mathematical Institute Belgrade, 2011.

[15] M. Kostić, Abstract Volterra Integro-Differential Equations, Taylor and Francis Group/CRS Press, accepted for publication.

[16] M. Kostić, Some contributions to the theory of abstract Volterra equations, Int. Journal Math. Anal. (Ruse) 5 (2011), 1529-1551.

[17] M. Kostić, $(a, k)$-regularized $C$-resolvent families: regularity and local properties, Abstr. Appl. Anal., vol. 2009, Art. ID 858242, 27 pages, 2009.

[18] M. Kostić, Abstract Volterra equations in locally convex spaces, Sci. China Math. 55 (2012), 1797-1825.

[19] M. Kostić, On a class of $(a, k)$-regularized $C$-resolvent families, Electron. J. Qual. Theory Differ. Equ. 94 (2012), 1-27.

[20] M. Kostić, C.-G. Li, M. Li, On a class of abstract time-fractional equations on locally convex spaces, Abstr. Appl. Anal., vol. 2012, Article ID 131652, 41 pages.

[21] M. Kostić, C.-G. Li, M. Li, S. Piskarev, On a class of time-fractional differential equations, Fract. Calc. Appl. Anal. 15 (2012), 639-668.

[22] M. Kostić, Perturbation theory for abstract Volterra equations, Abstr. Appl. Anal. vol. 2013, Art. ID 307684, 26 pp.

[23] M. Kostić, $(a, k)$-regularized $\left(C_{1}, C_{2}\right)$-existence and uniqueness families, Bull. Cl. Sci. Math. Nat. Sci. Math. in press.

[24] M. Kostić, Generalized well-posedness of abstract Volterra equations of non-scalar type, Ann. Acad. Rom. Sci. Ser. Math. Appl. accepted.

[25] K. Li, J. Peng, J. Jia, Cauchy problems for fractional differential equations with RiemannLiouville fractional derivatives, J. Funct Anal. 263 (2012), 476-510.

[26] C. Lizama, H. Prado, Fractional relaxation equations on Banach spaces, Appl. Math. Lett. 23 (2010), 137-142.

[27] A. Karczewska, C. Lizama, Solutions to stochastic fractional oscillation equations, Appl. Math. Lett. 23 (2010), 1361-1366.

[28] K. S. Miller, B. Ross, An Introduction to the Fractional Calculus and Differential Equations, John Wiley, New York, 1993.

[29] I. Podlubny, Fractional Differential Equations, Academic Press, New York, 1999.

[30] J. Prüss, Evolutionary Integral Equations and Applications, Vol. 87, Birkhäuser-Verlag, Basel, 1993.

[31] T.-J. Xiao, J. Liang, The Cauchy Problem for Higher-Order Abstract Differential Equations, Vol. 1701, Springer-Verlag, Berlin, 1998.

[32] T.-J. Xiao, J. Liang, Perturbations of existence families for abstract Cauchy problems, Proc. Amer. Math. Soc. 130 (2002), 2275-2285.

[33] T.-J. Xiao, J. Liang, Higher order abstract Cauchy problems: their existence and uniqueness families, J. London Math. Soc. 67(2) (2003), 149-164.

[34] J. Z. Zhang, Regularized cosine existence and uniqueness families for second order abstract Cauchy problems, Studia Math. 152 (2002), 131-145. 
${ }^{1}$ Department of Mathematics, Sichuan University, Chengdu 610064,

CHINA

E-mail address: cgang@scu.edu.cn

${ }^{2}$ Faculty of Technical Sciences, UNIVERSITY OF NOVI SAD,

Trg Dositeja Obradovića 6, 21125 NOVI SAD,

SERBIA

E-mail address: marco.s@verat.net

${ }^{3}$ Department of Mathematics, Sichuan University,

Chengdu 610064,

CHINA

E-mail address: mli@scu.edu.cn 